\title{
A CONTINUUM HAVING ITS HYPERSPACES NOT LOCALLY CONTRACTIBLE AT THE TOP
}

\author{
ALEJANDRO ILLANES
}

(Communicated by James E. West)

\begin{abstract}
For a continuum $X$, let $C(X)$ (resp. $2^{x}$ ) be the spaces of all nonempty subcontinua (resp. closed subsets) of $X$. In this paper we answer a question of Dilks by showing an example of a continuum $X$ such that if $\mathscr{H}=C(X)$ or $2^{x}$, then $\mathscr{H}$ does not have nonempty open subsets which are contractible in $\mathscr{H}$. In particular, $\mathscr{H}$ is not locally contractible at any of its points.
\end{abstract}

\section{INTRODUCTION}

A continuum is a compact, connected, nondegenerate metric space. The hyperspaces of a continuum $X$ are the spaces $2^{x}$ consisting of all nonempty closed subsets of $X$, and $C(X)$, consisting of the connected elements in $2^{x}$, each with the Hausdorff metric. If $U$ is a subset of a topological space $Y$, we say that $U$ is contractible in $Y$ if there exists a continuous function $F: U \times I \rightarrow$ $Y$ and there exists a point $y \in Y$ such that $F(u, 0)=u$ and $F(u, 1)=y$ for every $u \in U$.

In [4] the contractibility of hyperspaces is discussed in detail. More recent results may be found in [1], [2], [5-9]. In [3, Problem 111], appears the following question by Dilks: Is $C(X)$ or $2^{x}$ locally contractible at the point $X$ ? In this paper we give an example of a continuum $X$ such that if $\mathscr{H}=C(X)$ or $2^{x}$, then $\mathscr{H}$ does not have nonempty open subsets which are contractible in $\mathscr{H}$. In particular, $\mathscr{H}$ is not locally contractible at any of its points.

\section{AN AUXILIARY CONSTRUCTION}

Let $Q=[-1,1] \times[-1,1] \times \ldots$ with the metric $d(x, y)=\sum\left|x_{n}-y_{n}\right| /\left(2^{n}\right)$, where $x=\left(x_{1}, x_{2}, \ldots\right)$ and $y=\left(y_{1}, y_{2}, \ldots\right)$. Let $J=[-1,1]$, and let $\mathbb{N}=\{1,2, \ldots\}$. For $n \in \mathbb{N}$, define the projection $\Pi_{n}: Q \rightarrow J^{n}$ by $\Pi_{n}(x)=$ $\left(x_{1}, \ldots, x_{n}\right)$. Given $x, y \in Q$, define the segment joining $x$ and $y$ by $\overline{x y}=$ $\{t x+(1-t) y \in Q: t \in[0,1]\}$.

Received by the editors July 28, 1989 and, in revised form, January 22, 1990.

1980 Mathematics Subject Classification (1985 Revision). Primary 54B20.

Key words and phrases. Hyperspaces, contractibility. 
Given $n \in \mathbb{N}, A \subset J^{n} \times\{0\} \times \ldots$, and $p, q \in A$ define, for $m \in \mathbb{N}$, $A_{m}=\Pi_{n}(A) \times\{1 / m\} \times\{0\} \times \ldots, p_{m}=\left(\Pi_{n}(p), 1 / m, 0, \ldots\right) \in A_{m}, q_{m}=$ $\left(\Pi_{n}(q), 1 / m, 0, \ldots\right)$, and

$$
L_{m}= \begin{cases}\overline{p_{m} p_{m+1}} & \text { if } m \text { is even, } \\ \overline{q_{m} q_{m+1}} & \text { if } m \text { is odd. }\end{cases}
$$

Then we define $P(n, A, p, q)=A \cup\left(\bigcup\left\{A_{m} \cup L_{m}: m \in \mathbb{N}\right\}\right)$ and $N(n, A, p, q)$ $=\left\{\left(x_{1}, \ldots, x_{n},-x_{n+1}, x_{n+2}, \ldots\right) \in Q: x=\left(x_{1}, x_{2}, \ldots\right) \in P(n, A, p, q)\right\}$.

Since $A=\Pi_{n}(A) \times\{0\} \times \ldots$, we have that $P(n, A, p, q) \subset \Pi_{n}(A) \times[0,1] \times$ $\{0\} \times \ldots$. Then $\Pi_{n}(P(n, A, p, q))=\Pi_{n}(A)=\Pi_{n}(N(n, A, p, q))$. Notice that $A=\mathrm{Cl}_{Q}\left(\bigcup\left\{A_{m} \cup L_{m}: m \in \mathbb{N}\right\}\right)-\left(\bigcup\left\{A_{m} \cup L_{m}: m \in \mathbb{N}\right\}\right)$. So if $A$ is a continuum, then $P(n, A, p, q)$ and $N(n, A, p, q)$ are continua. Notice also that $P(n, A, p, q) \cap\left(J^{n} \times\{0\} \times \ldots\right)=A=N(n, A, p, q) \cap\left(J^{n} \times\{0\} \times \ldots\right)$.

For $n \in \mathbb{N}$ and $u \in J$, define $Q(n, u)=J^{n} \times\{u\} \times J \times \ldots$ If $m \in \mathbb{N}$ and $u \in(1 /(m+1), 1 / m)$, then $Q(n, u) \cap P(n, A, p, Q)=Q(n, u) \cap L_{m}$ is a point which separates $P(n, A, p, q)$.

\section{The EXAMPLE $X$}

Let $a=(-1,0, \ldots)$ and $b=(1,0, \ldots)$. For $n \in \mathbb{N}$, put $a_{n}=(-1+$ $\left.1 / 2^{n}, 0, \ldots\right)$ and $b_{n}=\left(1-1 / 2^{n}, 0, \ldots\right)$. Define

$$
\begin{aligned}
& A^{*}=\overline{a_{1} b_{1}} ; \\
& B_{1}=P\left(1, A^{*}, a_{1}, b_{1}\right) ; \\
& C_{1}=P\left(2, B_{1} \cup \overline{a_{2} b_{1}}, a_{2}, b_{1}\right), \\
& D_{1}=N\left(2, B_{1} \cup \overline{a_{1} b_{2}}, a_{1} . b_{2}\right) .
\end{aligned}
$$

In general, for $n \geq 2$, define $B_{n}=P\left(2 n-1, C_{n-1} \cup D_{n-1}, a_{n}, b_{n}\right) ; C_{n}=$ $P\left(2 n, B_{n} \cup \overline{a_{n+1} b_{n}}, a_{n+1}, b_{n}\right)$ and $D_{n}=N\left(2 n, B_{n} \cup \overline{a_{n} b_{n+1}}, a_{n}, b_{n+1}\right)$.

Then define $X=\mathrm{Cl}_{Q}\left(\bigcup\left\{B_{n}: n \in \mathbb{N}\right\}\right)$. Since $A^{*}$ is a continuum, then $B_{1}$, $C_{1}$, and $D_{1}$ are continua. It follows that each $B_{n}$ is a continuum. Furthermore $B_{1} \subset C_{1} \cap D_{1} \subset B_{2} \subset C_{2} \cap D_{2} \subset \ldots$ So $X$ is a continuum. We will prove some properties of $X$ :

$$
\Pi_{2 n+1}(X)=\Pi_{2 n+1}\left(C_{n} \cup D_{n} \cup \overline{a b}\right) \text { for all } n \in \mathbb{N} \text {. }
$$

Since $\overline{a_{n} b_{n}} \subset B_{n}$ and $\overline{a_{n} b_{n}} \rightarrow \overline{a b}$, we have that $\overline{a b} \subset X$. Then $C_{n} \cup D_{n} \cup$ $\overline{a b} \subset X$. If $m>n+1$, then $\Pi_{2 m-1}\left(B_{m}\right)=\Pi_{2 m-1}\left(C_{m-1} \cup D_{m-1}\right)$. This implies that

$$
\begin{aligned}
\Pi_{2 m-2}\left(B_{m}\right)= & \Pi_{2 m-2}\left(C_{m-1}\right) \cup \Pi_{2 m-2}\left(D_{m-1}\right) \\
= & \Pi_{2 m-2}\left(B_{m-1} \cup \overline{a_{m} b_{m-1}}\right) \cup \Pi_{2 m-2}\left(B_{m-1} \cup \overline{a_{m-1} b_{m}}\right) \\
& \subset \Pi_{2 m-2}\left(B_{m-1} \cup \overline{a b}\right) .
\end{aligned}
$$

So $\Pi_{2 m-3}\left(B_{m}\right) \subset \Pi_{2 m-3}\left(B_{m-1} \cup \overline{a b}\right)$. Then $\Pi_{2 n+1}\left(B_{m}\right) \subset \Pi_{2 n+1}\left(B_{m-1} \cup \overline{a b}\right)$. It follows that

$$
\Pi_{2 n+1}\left(B_{m}\right) \subset \Pi_{2 n+1}\left(B_{n+1} \cup \overline{a b}\right) .
$$




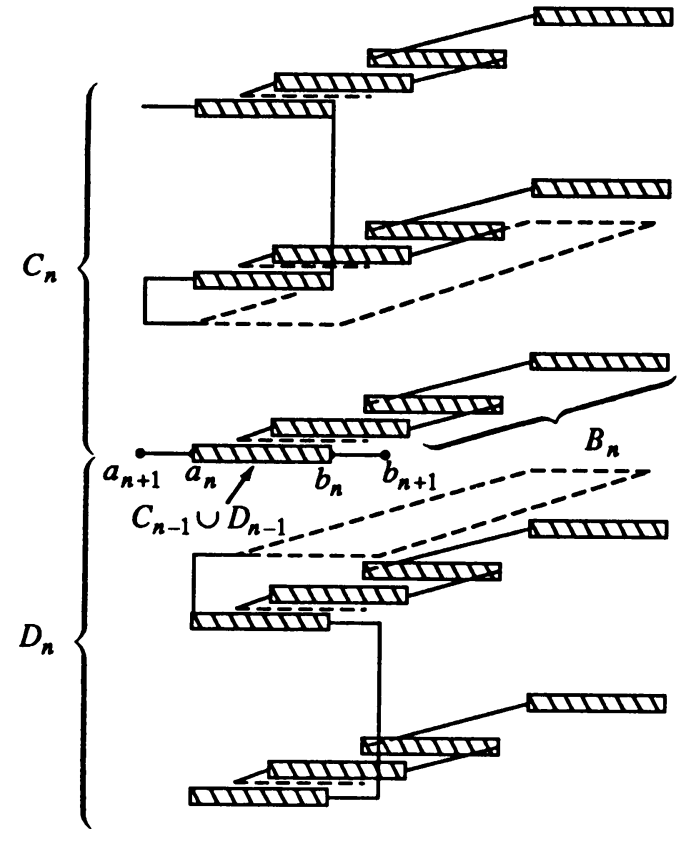

FigURe 1

But

$$
\begin{aligned}
& C_{n-1} \cup D_{n-1} J^{2 n-1} \times\{0\} \times \ldots \\
& B_{n} \subset J^{2 n-1} \times[0,1] \times\{0\} \times \ldots \\
& C_{n} \subset J^{2 n-1} \times[0,1] \times[0,1] \times\{0\} \times \ldots \\
& D_{n} \subset J^{2 n-1} \times[0,1] \times[-1,0] \times\{0\} \times \ldots
\end{aligned}
$$

$$
\Pi_{2 n+1}\left(B_{n+1}\right)=\Pi_{2 n+1}\left(C_{n} \cup D_{n}\right) ;
$$

$$
\Pi_{2 n+1}\left(\bigcup\left\{B_{m}: m>n\right\}\right) \subset \Pi_{2 n+1}\left(C_{n} \cup D_{n} \cup \overline{a b}\right) .
$$

Since $B_{1} \subset \cdots \subset B_{n} \subset C_{n} \cup D_{n}$ and $\Pi_{2 n+1}\left(C_{n} \cup D_{n} \cup \overline{a b}\right)$ is compact, we conclude that $\Pi_{2 n+1}(X)=\Pi_{2 n+1}\left(C_{n} \cup D_{n} \cup \overline{a b}\right)$.

(B) $\quad \Pi_{2 n}(X)=\Pi_{2 n}\left(B_{n} \cup \overline{a b}\right)$ for all $n \in \mathbb{N}$, and the maps $r_{1}: X \rightarrow$ $C_{n} \cup D_{n} \cup \overline{a b}$ and $r_{2}: X \rightarrow B_{n} \cup \overline{a b}$ given by $r_{1}(x)=\left(\Pi_{2 n+1}(x)\right.$, $0, \ldots)$ and $r_{2}(x)=\left(\Pi_{2 n}(x), 0, \ldots\right)$ are retractions.

Notice that $\Pi_{2 n}(X) \subset \Pi_{2 n}\left(C_{n}\right) \cup \Pi_{2 n}\left(D_{n}\right) \cup \Pi_{2 n}(\overline{a b}) \subset \Pi_{2 n}\left(B_{n} \cup \overline{a_{n+1} b_{n}}\right) \cup$ $\Pi_{2 n}\left(B_{n} \cup \overline{a_{n} b_{n+1}}\right) \cup \Pi_{2 n}(\overline{a b}) \subset \Pi_{2 n}\left(B_{n} \cup \overline{a b}\right)$. Since $C_{n} \cup D_{n} \cup \overline{a b} \subset J^{2 n+1} \times$ $\{0\} \times \ldots$, we have that $C_{n} \cup D_{n} \cup \overline{a b}=\Pi_{2 n+1}(X) \times\{0\} \times \ldots$. Then $r_{1}$ is a retraction. Similarly, $r_{2}$ is a retraction.

$$
B_{n} \cap \overline{a b}=\overline{a_{n} b_{n}} \text { for every } n \in \mathbb{N} \text {. }
$$


$B_{1} \cap \overline{a b}=P\left(1, A^{*}, a, b\right) \cap(J \times\{0\} \times \ldots)=A^{*}=\overline{a_{1} b_{1}}$. Suppose that $B_{n} \cap \overline{a b}=\overline{a_{n} b_{n}}$. Then $B_{n+1} \cap \overline{a b}=P\left(2 n+1, C_{n} \cup D_{n}, a_{n-1}, b_{n-1}\right) \cap\left(J^{2 n-1} \times\right.$ $\{0\} \times \ldots) \cap(J \times\{0\} \times \ldots)=\left(C_{n} \cup D_{n}\right) \cap\left(J^{2 n} \times\{0\} \times \ldots\right) \cap(J \times\{0\} \times \ldots)=$ $\left(\left(B_{n} \cup \overline{a_{n+1} b_{n}}\right) \cup\left(B_{n} \cup \overline{a_{n} b_{n+1}}\right)\right) \cap \overline{a b}=\overline{a_{n+1} b_{n+1}}$.

(D) Assume that $n \in \mathbb{N}, Y=C_{n} \cup D_{n} \cup \overline{a b}$ and $\mathscr{H}_{0}=C(Y)$ or $2^{Y}$. Then there do not exist $B \in \mathscr{H}_{0}, W$ open in $\mathscr{H}_{0}$ and $F: W \times I \rightarrow \mathscr{H}_{0}$ continuous such that $B \subset B_{n}, B \in W$, for each $A \in W, F(A, 0)=A, F(A, 1)=Y$ and, for every $A \in W$ and $s \leq t, F(A, s) \subset F(A, t)$.

Suppose that there exist such $B, W$, and $F$. Since $F(B, 0) \subset B_{n}$ and $F(B, 1)=Y \not \subset B_{n}$, there exists $t_{0}=\max \left\{t \in[0,1]: F(B, t) \subset B_{n}\right\}$ and $0 \leq t_{0}<1$. By $(C), a_{n+1}, b_{n+1} \notin B_{n}$ and, since $B_{n} \subset J^{2 n} \times\{0\} \times \ldots$, we have that $B_{n} \cap\left(\left(\left\{\Pi_{2 n}\left(a_{n+1}\right)\right\} \times J \times \ldots\right) \cup\left(\left\{\Pi_{2 n}\left(b_{n+1}\right)\right\} \times J \times \ldots\right)\right)=\varnothing$. So there exists $\delta>0$ such that $t_{0}+\delta / 2<1 ; \mathscr{B}=\left\{A \in \mathscr{H}_{0}: H(A, B)<\delta\right\} \subset W(H$ is the Hausdorff metric for $\left.2^{Q}\right)$ and, if $A \in \mathscr{B}$ and $t \in\left(t_{0}-\delta, t_{0}+\delta\right) \cap[0,1]$, then $F(A, t) \cap\left(\left(\left\{\Pi_{2 n}\left(a_{n+1}\right)\right\} \times J \times \ldots\right) \cup\left(\left\{\Pi_{2 n}\left(b_{n+1}\right\} \times J \times \ldots\right)\right)=\varnothing\right.$.

We will prove that $F\left(B, t_{0}+\delta / 2\right) \subset C_{n}$. Recall that

$$
\begin{aligned}
C_{n} & =P\left(2 n, B_{n} \cup \overline{a_{n+1} b_{n}}, a_{n+1}, b_{n}\right) \\
& =B_{n} \cup \overline{a_{n+1} b_{n}} \cup\left(\bigcup\left\{A_{m} \cup L_{m}: m \in \mathbb{N}\right\}\right)
\end{aligned}
$$

where $A_{m}=\left(\Pi_{2 n}\left(B_{n} \cup \overline{a_{n+1} b_{n}}\right)\right) \times\{1 / m\} \times\{0\} \times \ldots$;

$$
\begin{aligned}
L_{m} & = \begin{cases}\overline{p_{m} p_{m+1}} & \text { if } m \text { is even } \\
\overline{q_{m} q_{m+1}} & \text { if } m \text { is odd, }\end{cases} \\
p_{m} & =\left(\Pi_{2 n}\left(a_{n+1}\right), 1 / m, 0, \ldots\right), \\
q_{m} & =\left(\Pi_{2 n}\left(b_{n}\right), 1 / m, 0, \ldots\right) .
\end{aligned}
$$

Since $B \subset B_{n} \subset J^{2 n} \times\{0\} \times \ldots$, we have that $B=\Pi_{2 n}(B) \times\{0\} \times \ldots$ For each $m \in \mathbb{N}$, put $E_{m}=\Pi_{2 n}(B) \times\{1 / m\} \times\{0\} \times \cdots \subset A_{m}$. Then $E_{m} \in \mathscr{H}_{0}$ and $E_{m} \rightarrow B$, so there exists $M \in \mathbb{N}$ such that $H\left(B, E_{m}\right)<\delta$ for all $m \geq M$.

Let $m$ be an even number with $m \geq M$. We will show that

$$
T=F\left(E_{m}, t_{0}+\delta / 2\right) \subset C_{n} .
$$

Choose $u \in(1 /(m+1), 1 / m)$. Then $Q(2 n, u) \cap C_{n}=Q(2 n, u) \cap \overline{p_{m} p_{m+1}}$. Since $\left\{F\left(E_{m}, s\right) \in 2^{Y}: 0 \leq s \leq t_{0}+\delta / 2\right\}$ is an order arc in $2^{Y}$ from $F\left(E_{m}, 0\right)$ to $T$, then [4, Theorem 1.8] each component of $T$ intersects $E_{m} \subset \Pi_{2 n}\left(B_{n}\right) \times\{1 / m\} \times$ $\{0\} \times \ldots$. Thus $S=T \cup\left(\Pi_{2 n}\left(B_{n}\right) \times\{1 / m\} \times\{0\} \times \ldots\right)$ is connected. Since $\overline{p_{m} p_{m+1}} \subset\left\{\Pi_{2 n}\left(a_{n+1}\right)\right\} \times J \times \ldots, B_{n} \subset J^{2 n} \times\{0\} \times \ldots$, and $a_{n+1} \notin B_{n}$, we have that $S \cap \overline{p_{m} p_{m+1}}=\varnothing$. On the other hand, $S \subset Y=C_{n} \cup D_{n} \cup \overline{a b}$ and $D_{n} \cup \overline{a b} \subset$ $J^{2 n} \times[-1,0] \times\{0\} \times \ldots$, so $S \cap Q(2 n, u)=S \cap Q(2 n, u) \cap C_{n}=S \cap Q(2 n, u) \cap$ $\overline{p_{m} p_{m+1}}=\varnothing$. Furthermore $S$ is connected and $E_{m} \subset S \cap J^{2 n} \times(u, 1] \times J \times \ldots$. 
Then $S \subset\left(J^{2 n} \times(u, 1] \times J \times \ldots\right) \cap Y \subset C_{n}$. Thus $F\left(E_{m}, t_{0}+\delta / 2\right) \subset C_{n}$ for all $m \geq M$ with $m$ even. Hence $F\left(B, t_{0}+\delta / 2\right) \subset C_{n}$.

In a similar way it may be proved that

$$
F\left(B, t_{0}+\delta / 2\right) \subset D_{n} .
$$

Then $F\left(B, t_{0}+\delta / 2\right) \subset D_{n}$. Then $F\left(B, t_{0}+\delta / 2\right) \subset C_{n} \cap D_{n}=C_{n} \cap\left(J^{2 n} \times\{0\} \times\right.$ $\ldots) \cap D_{n}=\left(B_{n} \cup \overline{a_{n+1} b_{n}}\right) \cap\left(B_{n} \cup \overline{a_{n} b_{n+1}}\right)=B_{n}$. Therefore $F\left(B, t_{0}+\delta / 2\right) \subset B_{n}$. This contradicts the choice of $t_{0}$ and proves $(D)$.

$$
C(X)=\mathrm{Cl}_{c(x)}\left(\bigcup\left\{C\left(B_{n}\right): n \in \mathbb{N}\right\}\right),
$$

and

$$
2^{x}=\mathrm{Cl}_{2^{x}}\left(\bigcup\left\{2^{B n}: n \in \mathbb{N}\right\}\right) .
$$
by

For $n \in \mathbb{N}$, take the natural retraction $f: \overline{a b} \rightarrow \overline{a_{n} b_{n}}$ and define $r: X \rightarrow B_{n}$

$$
r(x)= \begin{cases}f\left(r_{2}(x)\right) & \text { if } r_{2}(x) \in \overline{a b}, \\ r_{2}(x) & \text { if } r_{2}(x) \in B_{n},\end{cases}
$$

with $r_{2}$ as in $(B)$. Then $r$ is a retraction such that $D(x, r(x)) \leq 1 /\left(2^{n-1}\right)$ for every $x \in X$. Hence, for each $B \in 2^{x}, H(B, r(B)) \leq 1 /\left(2^{n-1}\right)$.

If $\mathscr{H}=C(X)$ or $2^{x}$, then $\mathscr{H}$ does not have nonempty open subsets which are contractible in $\mathscr{H}$.

Suppose that there exist an open nonempty subset $U$ of $\mathscr{H}$, a continuous function $G: U \times I \rightarrow \mathscr{H}$, and $A_{0} \in \mathscr{H}$ such that $G(A, 0)=A$ and $G(A, 1)=$ $A_{0}$ for every $A \in U$. Then the function $K: U \times I \rightarrow \mathscr{H}$ given by $K(A, t)=$ $\bigcup\{G(A, s): 0 \leq s \leq t\}$ is continuous [4, Lemma 16.3]. Let $\alpha:[1 / 2,1] \rightarrow \mathscr{H}$ be a continuous function such that $\alpha(1 / 2)=A_{0}, \alpha(1)=X$ and $\alpha(s) \subset \alpha(t)$ if $s \leq t$. Choose $n \in \mathbb{N}$ such that $U \cap 2^{B n} \neq \varnothing$. Let $Y=C_{n} \cup D_{n} \cup \overline{a b}$, $\mathscr{H}_{0}=2^{Y} \cap \mathscr{H}$, and $W=U \cap \mathscr{H}_{0}$. Fix $B \in W \cap 2^{B n}$. Define $F: W \times I \rightarrow \mathscr{H}_{0}$ by:

$$
F(A, t)= \begin{cases}r_{1}(K(A, 2 t)) & \text { if } 0 \leq t \leq 1 / 2, \\ r_{1}(K(A, 1) \cup \alpha(t)) & \text { if } 1 / 2 \leq t \leq 1,\end{cases}
$$

where $r_{1}$ is the retraction defined in $(B)$ and $r_{1}(Z)$ means the image of $Z$ under $r$. Properties of $B, W$, and $F$ contradict property $(D)$, and the contradiction completes the proof of $(F)$.

Added in proof. The question answered in this paper has also been answered by Hisao Kato, On local contractibility at $X$ in hyperspaces $C(X)$ and $2^{X}$, Houston J. Math. 15 (1989), 363-370. 


\section{BIBLIOGRAPHY}

1. D. W. Curtis, Application of a selection theorem to hyperspace retractibility, Canad. J. Math. 37 (1985), 747-759.

2. A. Illanes, Arc-smoothness and contractibility in Whitney levels, Proc. Amer. Math. Soc. (to appear).

3. W. Lewis, Continuum theory problems, Topology Proc. 8 (1983), 361-394.

4. S. B. Nadler, Jr., Hyperspaces of sets, Marcel Dekker, New York, 1978.

5. T. Nishiura and C. J. Rhee, Contractibility of the hyperspace of subcontinua, Houston J. Math. 8 (1982), 119-127.

6. $\ldots$, An admissible condition for contractible hyperspaces, Topology Proc. 8 (1983), 303314.

7. C. J. Rhee, On a contractible hyperspace condition, Topology Proc. 7 (1982), 147-155.

8. _ Contractible hyperspace of subcontinua, Kyunpook Math. J. 24 (1984), 143-154.

9. __ Obstructing set for hyperspace contraction, Topology Proc. 10 (1985), 159-173.

Instituto de Matematicas, Circuito Exterior, Ciudad Universitaria, Mexico 04510, Distrito Federal, Mexico 